\title{
Metastasis Suppressors and the Tumor Microenvironment
}

\author{
Thomas M. Bodenstine • Danny R. Welch
}

Received: 19 December 2007 / Accepted: 3 January 2008 / Published online: 31 January 2008

(C) Springer Science + Business Media B.V. 2008

\begin{abstract}
The most dangerous attribute of cancer cells is their ability to metastasize. Throughout the process of metastasis, tumor cells interact with other tumor cells, host cells and extracellular molecules. This brief review explores how a new class of molecules - metastasis suppressors regulate tumor cell-microenvironmental interactions. Data are presented which demonstrate that metastasis suppressors act at multiple steps of the metastatic cascade. A brief discussion for how metastasis suppressor regulation of cellular interactions might be exploited is presented.
\end{abstract}

Keywords Metastasis suppressor $\cdot \mathrm{Nm} 23 \cdot \mathrm{RECK}$. BRMS1 · KISS1 · TIMP · E-cadherin · MKK4 · KAI1 · Adhesion · Invasion · MMP - Intravasation · Cohesion · Motility $\cdot$ Endothelium $\cdot$ Angiogenesis $\cdot$ Review

T. M. Bodenstine $\cdot$ D. R. Welch $(\bowtie)$

Department of Pathology, University of Alabama at Birmingham, VH-G019,

Birmingham, AL 35294-0019, USA

e-mail: danwelch@uab.edu

D. R. Welch

Department of Cell Biology,

University of Alabama at Birmingham,

Birmingham, AL, USA

D. R. Welch

Department of Pharmacology and Toxicology,

University of Alabama at Birmingham,

Birmingham, AL, USA

D. R. Welch

Comprehensive Cancer Center,

University of Alabama at Birmingham,

Birmingham, AL, USA

D. R. Welch

National Foundation for Cancer Research-Center for Metastasis

Research, University of Alabama at Birmingham,

Birmingham, AL, USA

\section{Introduction}

The metastatic cascade describes the process where neoplastic cells in a primary tumor leave their initial location of growth, travel to nearby or distant secondary sites and proliferate into a macroscopic, clinically relevant mass. The process involves intrinsic (i.e., genetic) as well as extrinsic (i.e., tumor cell-microenvironmental signals) factors. In this brief review, our objective is to highlight how metastatic cell interactions with the tumor microenvironment are affected by, or regulated by, metastasis suppressors at various steps of the metastatic cascade. Because of space limitations, we will refer readers to several excellent reviews and primary literature for details on specific topics.

The process of hematogenous and lymphatic metastasis begins when tumor cells attain the ability to break away and grow independently from a primary tumor while migrating through the complex network of proteins, proteoglycans and collagens of the extracellular matrix [1,2]. These cells then intravasate into the blood or lymphatic vessels where they survive the shear stress of transport [3, 4], resist anoikis [5] and evade immune surveillance [6, 7]. Following vessel transport, metastatic cells may become lodged in capillary beds due to their size or the size of emboli (both homotypic or heterotypic), or adhere to integrins and other, as yet only partly defined, receptors on organ-specific endothelial cells [8-12]. In response to chemoattractants [13], extravasation from vessels at a secondary location follows [14], but is not absolutely essential [15] to proceed to subsequent steps. Following extravasation, metastatic cells must then complete the most crucial, and perhaps most selective, step of the metastatic cascade, proliferation at the secondary site [16-19]. It is important to note that some metastatic cells arrive at a secondary site by means other than those described above: migration across body cavities and peri-vascular transport have been reported for some metastatic cell types. However, regardless of the means of 
transport or migration, metastasis is defined by a crucial hallmark of metastatic disease: establishment of a cell mass at a site discontinuous from the primary tumor [18, 19].

Even from this outline, it is clear that tumor cell interactions with the microenvironment are crucial at many steps throughout the metastatic process and contribute to a high level of complexity to what we know about metastasis. Cancer cells are not only dependent on genetic alterations that distinguish them from their normal counterparts, but are also sensitive to extracellular factors that may or may not allow them to metastasize. Tumor cells alter the microenvironment directly (e.g., proteolytic cleavage of the ECM) or indirectly (e.g., induction of nearby cells to secrete proteases) [20]. Conversely, tumor cells can be impacted by stromal cells (e.g., lymphatic or vascular) at each step in the cascade. Thus, it is important for researchers to not only focus on the genetic mutations, deletions or chromosomal translocations within a tumor cell, but also recognize that stromal factors are present and intimately involved in the process. What we learn from these interactions will help to provide more potent therapies targeting metastasis.

We begin with a simple premise - i.e., that some, if not most, of the genetic changes occurring within cancer cells either manipulate, or are manipulated by, the various microenvironments in which the cells find themselves. The breadth of that hypothesis cannot be covered in the space allowed. So, we will focus this review exclusively upon how changes in the expression of a new class of genes, metastasis suppressors, alter tumor microenvironmental changes.

\section{Metastasis Suppressors}

A metastasis suppressor is defined as a molecule whose expression results in the inhibition of a cancer cell's ability to metastasize while having little or no effect on primary tumor growth (reviewed in [21, 22]). This family was first described in the mid-1980's with the discovery of Nm23. Since then, more than 20 metastasis suppressors (Table 1) have been reported with more expected to be discovered in years to come. However, although much has been learned, the detailed molecular mechanisms of how these genes act have not been fully elucidated. Their actions are diverse and include the regulation of angiogenesis, extracellular matrix remodeling, transcription regulation and signal transduction. The order of discussion roughly follows the

Table 1 Summary of metastasis suppressors and proposed mechanisms of action

\begin{tabular}{|c|c|c|}
\hline Suppressor & Function(s) & $\begin{array}{l}\text { Types of tumor cell- } \\
\text { microenvironment } \\
\text { interactions affected }\end{array}$ \\
\hline BRMS1 & $\begin{array}{l}\text { Chromatin remodeling; transcription regulation; reduces } \\
\text { phosphoinositide signaling; restores gap junctional intercellular } \\
\text { communication }\end{array}$ & Survival in transport, colonization \\
\hline $\begin{array}{l}\text { Cadherins E-Cadherin } N \text { - } \\
\text { Cadherin Cadherin- } 11\end{array}$ & Cell-cell or cell-matrix adhesion; homotypic cohesion & EMT, invasion \\
\hline Caspase-8 & Induce apoptosis/anoikis & Survival in transport \\
\hline CD44 & Cell-cell or cell-matrix adhesion; bind hyaluronic acid and osteopontin & Migration \\
\hline DCC & Cytoplasmic architecture; MAPK signaling & Motility, invasion \\
\hline DLC1 & Signaling via Rho-GTPase; cytoskeletal architecture & Migration, invasion \\
\hline $\operatorname{Drg} 1$ & Unknown & \\
\hline Gelsolin & Actin regulatory molecule; cytoskeletal architecture & Migration \\
\hline KAI1 & Bind DARC; integrin interaction; EGFR desensitization & Intravasation, survival in transport \\
\hline KISS1 (kisspeptins) & Ligand for G-protein coupled receptor & Colonization \\
\hline MKK4 MKK7 p38 & Stress-activated MAPK signaling & Migration, colonization \\
\hline $\mathrm{Nm} 23$ & Inhibition of ras signaling; histidine kinase regulation; NDP kinase & Migration, colonization \\
\hline OGR1 & G-protein coupled receptor signaling & Migration, colonization \\
\hline RhoGDI2 & Cytoskeletal architecture; endothelin and neuromedin U signaling & Migration, colonization \\
\hline RKIP & Raf-MEK signaling; cytoskeletal organization & Migration, invasion \\
\hline RRM1 & PI3K signaling from focal adhesions; cytoskeletal architecture; adhesion & Motility, invasion \\
\hline SSeCKs & $\begin{array}{l}\text { Scaffold for protein kinases; Src signaling, PKC signaling, } \\
\text { Rho signaling; VEGF secretion }\end{array}$ & Angiogenesis, migration \\
\hline TIMPs & Inhibition of metalloproteinases; signaling & $\begin{array}{l}\text { Angiogenesis, migration, invasion, } \\
\text { survival in transport, colonization }\end{array}$ \\
\hline
\end{tabular}

Not all of the metastasis suppressors listed in this table are discussed in detail. Readers are referred to reviews on metastasis suppressors mentioned in the text for additional details 
steps of the metastatic cascade impacted by metastasis suppressors.

\section{E-Cadherin}

E (epithelial)-cadherin is one of the most studied proteins in cancer progression and metastasis. A transmembrane glycoprotein, E-cadherin binds homotypically to E-cadherin on neighboring cells through $\mathrm{Ca}^{+2}$-dependent extracellular domains. E-cadherin anchors to actin filaments in the cytoplasm through structural interactions with $\alpha$ - and $\beta$ catenin. This acts to promote cellular polarity in epithelial layers by cytoskeletal organization. Regulation of E-cadherin occurs via multiple mechanisms, including receptor tyrosine kinases, transforming growth factor beta (TGF- $\beta$ ) and NFKB signaling (reviewed in [23]) as well as DNA methylation $[24,25]$.

Downregulation of E-cadherin expression is known to induce (or is a consequence of) an epithelial-to-mesenchymal transition (EMT), a process in which epithelial cells revert to a more motile mesenchymal phenotype believed to play a major role in the invasion and metastasis of cancer cells [26-29]. The loss of surface E-cadherin protein, resulting in a loss of polarity and reduced association with other epithelial cells, is a hallmark of EMT. EMT itself is a known cellular process in the maturing embryo where cells migrate to developing regions of an organism, but induction of EMT in adult cells is believed to play a negative role when cancer cells employ this process to enhance their invasiveness [26-29].

Shortly after the initial discovery and description of the cadherin family, a number of studies reported expression of E-cadherin to be inversely correlated to invasive qualities in cancer cells and that transfection of E-cadherin into invasive cell lines reduced their malignancy [30]. A continually growing cohort of clinical and experimental data confirms the anti-metastatic capabilities of E-cadherin [31] and significance of EMT [32, 33].

As cells growing in an epithelial layer begin to lose contact with themselves (cohesion) they often begin to produce cellular protrusions through reorganization of the cytoskeleton and plasma membrane that form new contacts with the surrounding microenvironment $[1,2,34]$. These new adhesive contacts promote the migration of cells through the extracellular matrix and plays on a theme that as cancer cells progress to a more metastatic phenotype, they become more independent and less reliant on other cells for survival signals and growth factors (loss of gap junction communication, anoikis, etc.). By continuing to further delineate the multiple molecular pathways involved in the induction of EMT and loss of E- cadherin, a better understanding of how cells may be inhibited in these processes will lead to more adept antimetastatic therapies.

However, it is still not certain that EMT is required for metastasis [35-37]. For example, recent data have identified different mechanisms by which tumor cells can invade surrounding tissues and extracellular matrices (reviewed in [1]). Many carcinomas migrate as clusters of cells, suggesting that they have maintained vestigial epithelialepithelial interactions (perhaps, though not directly tested to our knowledge, maintaining E-cadherin expression). The EMT-based motility refers to migration of individual cells in a manner dependent upon proteolysis; whereas, individual cell movement independent upon proteases the so-called epithelial-amoeboid transition - is becoming increasingly recognized.

\section{SSeCKS}

Src Suppressed C Kinase Substrate (SSeCKS) was discovered using PCR based subtractive hybridization in NIH3T3 mouse fibroblasts and found to be suppressed by oncogenic forms of src, ras, fos, and myc [38]. SSeCKS mRNA was found to accumulate in non-diving cells, while $S S e C K S$ mRNA was suppressed in dividing or transformed cells. SSeCKS protein is phosphorylated by protein kinase C (PKC) and involved in cytoskeletal architecture by binding F-actin and mediating signaling pathways [38, 39]. SSeCKS was first described as a metastasis suppressor when its expression was able to inhibit the ability of MatLyLu rat prostate cancer cells to metastasize to lungs in nude mice after flank injection but had minimal effect on overall tumorigenicity of the cells. This experimental evidence was supported by clinical data showing SSeCKS expression in benign and well differentiated prostate carcinomas, but exhibited loss of expression in highly aggressive and undifferentiated prostate lesions [40].

The molecular mechanisms reported for SSeCKS, thus far, involve a complex signaling activation sequence that can be initiated by extracellular factors. The binding of growth factors and adhesion of integrins to proteins of the extracellular matrix have both been shown to activate multiple signaling and phosphorylation cascades, leading to cytoskeletal reorganization and cell migration mediated through signaling molecules such as $\mathrm{Src}$ and PI3K. SSeCKS, in its dephosphorylated state, acts as a scaffolding protein where it binds signaling molecules such as PKC, PKA, calmodulin and cyclins. Upon mitogenic signaling, SSeCKS is phosphorylated and translocates to the perinuclear membrane releasing its bound signaling molecules to mediate changes within a cell [41]. 
Most interesting may be SSeCKS involvement in focal adhesions and their combinatorial roles on cytoskeletal rearrangement. Focal adhesions are formed at the plasma membrane when receptors or integrins bind extracellular matrix proteins and induce clustering of actin and other protein complexes at these sites. Focal adhesion kinase (FAK) is a protein tyrosine kinase recruited to focal adhesions. Phosphorylation of SSeCKS through stimulation by multiple growth factors has been shown to be FAK dependent through mediation of an unidentified FAK induced kinase [42]. This FAK-dependent phosphorylation of SSeCKS not only induces its release of signaling molecules, but reduces its F-actin binding capability. Thus, SSeCKS acts to sequester growth factors and binds F-actin when in its dephosphorylated state during G0 and early G1 phases. But upon mitogenic signaling SSeCKS relinquishes actin binding and allows induction of signaling cascades leading to changes in the cytoskeleton.

The effects of SSeCKS as a metastasis suppressor can be best appreciated if SSeCKS acts as a regulator of cytoskeleton reorganization and migration. Lack of expression or loss of function, could promote the migration of primary tumor cells to a secondary site by allowing a more motile phenotype.

\section{$\mathrm{Nm} 23$}

Nm23 (non-metastatic clone 23) gene was discovered by screening K1735 murine melanoma cells with varying metastatic capability in an attempt to identify metastasis related genes differentially expressed between high and low metastatic cells [43]. Two of the poorly metastatic cell lines had a 10-fold higher expression of Nm23 mRNA than the other five related highly metastatic lines. Transfection of $N m 23$ into the highly metastatic cell clones still allowed tumor growth but metastasis was inhibited, thereby fulfilling the definition of a metastasis suppressor. Prior to the discovery of $\mathrm{Nm} 23$, it was shown that when rat embryo fibroblasts (REFs) were transformed with the human oncogene c-Ha-ras or co-transfected with c-Ha-ras and the adenoviral type 2 Ela gene, both groups formed tumors when injected subcutaneously (s.c.) into athymic mice. However only c-Ha-ras transfected cells formed metastatic lung nodules, while the E1a co-transfected cells formed significantly fewer lung metastases [44]. Subsequently, it was found that $\mathrm{Nm} 23$ was upregulated in the REF cells by the E1a gene, consistent with a role of $\mathrm{Nm} 23$ as a metastasis suppressor. The anti-metastatic activities of $\mathrm{Nm} 23$ have since been demonstrated in multiple cancer models (reviewed in $[45,46])$ and $\mathrm{Nm} 23$ expression inhibits invasion, motility, and anchorage independent growth in vitro [47]. Nm23-H1 (human isoform 1; there are now eight known human Nm23 isoforms) has been correlated to clinical data in multiple tumor types [45, 46]. Low levels of Nm23 mRNA expression have been associated with good patient prognosis and reduced metastases. Although the preponderance of evidence supports $\mathrm{Nm} 23-\mathrm{H} 1$ as a metastasis suppressor, conflicting clinical reports suggest the possibility that the actions of Nm23-H1 are cell and tissue type specific [48]. These findings may be due to multiple isoforms, homologs and other regulatory mechanisms of $\mathrm{Nm} 23$ (reviewed in [49, 50]). Efforts to characterize the tumorigenic and metastatic implications of other Nm23 homologs are currently underway [47].

Data suggesting possible Nm23 interactions with the microenvironment have been reported, but a precise mechanism for how Nm23 affects cell migration and motility still remain unclear. Numerous reports link Nm23-H1 with the Ras family of proteins. Nm23-H1 was shown to bind and inhibit the activity of Tiam1, a guanine exchange factor (GEF) downstream of Ras that activates Rac1, a small GTP binding protein that leads to proliferation and migration by cytoskeletal reorganization [51]. Data also exist correlating involvement in the upregulation of matrix MMP-9 in B-cell lines when Nm23-H1 function was altered by the Epstein Barr virus latent protein EBNA3C [52]. Conversely, transfection of Nm23 in the L9981 human non-small cell lung cancer cell line decreased levels of MMP2 [53].

More recent data shows a novel link in the Nm23-H1 mediated suppression of metastasis. Using gene expression microarrays, comparison of cancer cells transfected to overexpress wild-type Nm23-H1 with MDA-MB-435 expressing mutant forms of Nm23-H1, nine genes were down-regulated by wild-type Nm23 and correlated to human breast cancer cohorts [54]. Specifically, EDG2 (endothelial differentiation, lysophosphatidic acid G-protein-coupled receptor 2), a lysophosphatidic acid (LPA) receptor restored motility when overexpressed in Nm23-H1 suppressed cells. Knock down of EDG2 by siRNA in metastatic cells reduced their motility. These findings fit well with previous data involving Nm23$\mathrm{H} 1$ and G-proteins in that LPA is a potent signaling molecule which is mitogenic and activates the GTPase Rho. The latter initiates cytoskeletal reorganization and induces cellular migration. Further examination of these findings and characterization of the regulation of EDG2 by Nm23 may provide new insight into a more detailed description of how $\mathrm{Nm} 23$ controls cellular responses from the microenvironment.

\section{TIMPs}

The tissue inhibitors of metalloproteinases are a family of four endogenously expressed, secreted protease inhibitors, TIMP1-4, that inhibit the function of MMPs (matrix 
metalloproteinases). Due to their nature of action, TIMPs can inhibit the remodeling and plasticity of the extracellular environment caused by MMPs. In the context of metastasis, early reports showed human prostate tissue samples exhibited higher expression levels of MMP2 and MMP9 and reduced TIMP levels in malignant tissues compared to normal prostate and breast tissue. Experimental studies confirmed clinical data showing that levels of TIMP1 correlated inversely to metastatic potential in colon carcinoma cell lines with differing metastatic potential [55]. Transfection of TIMP2 into human lung carcinoma cell lines decreased colony formation in soft agar and invasion in Matrigel [56]. The anti-invasive properties from expression or treatment with TIMPs has continued to be observed in models of cancer and correlates to their role as metastasis suppressors.

However, the balance of MMPs and TIMPs is not as straightforward as originally anticipated. While many studies have shown a decrease in TIMPs in metastatic disease some experimental models suggest TIMPs increase cancer cell invasion. At the forefront of these discrepancies is the complexity of the MMP2, TIMP2, MT1-MMP (MMP14) axis. TIMP2 binds MMP2 in its latent form. This complex can then associate with MT1-MMP and facilitate the activation of MMP2, thus providing a role for TIMP2 in the promotion and activity of MMP2 (reviewed in [57]).

It is clear that TIMPs can act to suppress the invasive effects of MMPs in both clinical samples and experimental models; however, there is now believed to be a higher level complex system involving cooperation of various MMPs and TIMPs. So, their actions must be further studied to determine the exact pathways involved. In addition, it is likely that there is a high involvement of stromal cells influencing the actions of MMPs and TIMPs and these interactions must be considered when examining levels of MMPs/TIMPs in cancer cells and clinical samples.

\section{RECK}

RECK (reversion-inducing cysteine rich protein with Kazal motifs) was originally identified using a cDNA library screen on v-Ki-RAS transformed fibroblasts where it exerted a flat morphology phenotype (i.e., revertant) [58]. RECK mapped to chromosome 9, and its protein product is a membrane bound glycoprotein capable of inhibiting the secretion of MMP9, invasion and metastasis [58]. RECK is downregulated in many oncogenic transformed cell lines, while forced expression of RECK in metastatic cell lines leads to a reduction in invasion and metastasis [59]. RECK is expressed in most normal cells and proven as a prognostic marker for patient outcome in hepatocellular, prostate, breast, colorectal and non-small cell lung carcinomas [59-61]. RECK also inhibits the activity of MMP-2 and membrane-type (MT)-MMP1 [59, 62]. This suggests a role for RECK in regulating interactions of MMPs, which have numerous roles in most steps of the metastatic cascade $[20,63]$.

The transcriptional regulation of RECK has been investigated and involves the $\mathrm{Sp} 1$ family of transcription factors, histone deacetylation and methylation and are discussed elsewhere [59, 64-66]. What are the direct implications of RECK dependent downregulation on activity of MMPs, and how does this relates to tumor progression and metastasis? Building evidence links MMP9 and MMP-2 to angiogenesis and invasion. When HT1080 fibrosarcoma cells were transfected to express RECK and injected into nude mice, no changes in tumor size were noted. However as tumors grew, RECK expressing tumor vessel density and vessel sprouting were reduced. Both changes lead to longer survival and less vascularized tumors [67]. This was confirmed in clinical samples of human esophageal squamous cell carcinoma. RECK expression decreased during tumor progression and was inversely correlated to lymph node metastases, vascular endothelial growth factor (VEGF) and endoglin (CD105) expression [68]. RECK, therefore, appears to be a regulator of angiogenesis, perhaps through MMP regulation.

\section{KAI1}

KAII/CD82 was discovered after its ability to suppress metastasis when transfected into the prostate cancer cell line AT6.1 and was mapped to chromosome 11p11.2 [69]. The expression of KAII is downregulated in numerous cancer cell lines and its metastasis suppressive capability has been strongly correlated with clinical data from a variety of human cancers including non-small cell lung carcinoma, pancreatic, bladder and breast cancers [70, 71].

The KAIl protein is a member of the tetraspanin superfamily subject to post translational modifications including glycosylation and palmitoylation. Since its discovery KAI1 has been shown to interact with many proteins, including other tetraspanins, integrins, growth factor receptors, uPAR, KITENIN and protein kinase $\mathrm{C}$ (PKC), while its transcriptional regulation is affected by $\mathrm{NF} \kappa \mathrm{B}, \mathrm{p} 53, \beta-$ catenin and other transcriptional factors downstream of MAPK pathways (reviewed in [70]). Collectively, expression of KAI1 leads to a decrease in cellular motility and has been shown to induce apoptosis and reduce anchorage independent growth in some models. Additionally, KAIl has been demonstrated to decrease protein and mRNA levels of MMP9, while increasing TIMP1 levels when transfected into the human lung carcinoma cell line H1299 [72]. All of 
these activities are likely to be involved in the regulation of cell migration, but a unified theory of how KAI1 inhibits metastasis, or where in the metastatic cascade KAI1 acts, had yet to be determined.

An important discovery in the function of KAI1 was recently made. Using a normal human prostate cDNA library, a yeast two-hybrid screen with KAII cDNA identified KAI1 interactors, including strong binding affinity with Duffy antigen receptor for chemokines (DARC) [73]. DARC is highly expressed in vascular and lymphatic endothelium of the prostate while little DARC was found on the epithelium and stroma. Cell-cell binding assays showed KAI1 on AT6.1 prostate cancer cells were able to bind when overlain onto endothelial cells expressing DARC. KAI1-expressing cells binding DARC had reduced DNA synthesis and were made senescent in prostate, breast and lung cancer cell lines. Similar growth inhibition and senescence were not observed for KAI1-expressing cells in Darc $^{-/-}$mice, concomitant with failure to reduce metastasis. Based upon these findings, KAI1 expressing cells are rendered incapable of proceeding or are eliminated when leaving the primary tumor (i.e., intravasation into the blood or lymphatic vessels).

\section{BRMS1}

The observation that loss of genetic material on chromosome 11 occurs in many metastatic breast carcinomas lead to the discovery of Breast Cancer Metastasis Suppressor 1 (BRMS1). Microcell-mediated transfer of chromosome 11 into MDA-MB-435 suppressed metastasis to the lungs and regional lymph nodes by $>95 \%$ while minimally affecting orthotopic growth in the mammary fat pad. Differential display identified BRMS1, which mapped to 11q13.111q13.2. Structural analysis of the encoded protein identified multiple coiled coil domains, an imperfect leucine zipper motif, and putative phosphorylation and nuclear localization signal sites, suggesting protein-protein interactions and possible transcriptional regulation [74]. BRMS1 metastasis suppressing ability has extended to multiple xenograft and syngeneic models [75, 76].

The clinical correlates of BRMS1 are more complex. Since antibodies recognizing BRMS1 have only recently been developed, most studies have relied on mRNA expression. The correlations have been mixed, showing loss of BRMS1 mRNA correlating with increased tumor grade and poor prognosis [77] or high levels of BRMS1 mRNA correlated with better prognosis and patient outcome [78-80]. In other studies, a lack of correlation exists between BRMS1 mRNA levels and axillary lymph node metastases in breast cancer samples [81]. In the first, and as-yet only, published study examining BRMS1 protein, reduced BRMS1 expression correlated to worsened disease free survival in a subset of cancer patients with overexpression of $\mathrm{Her} 2$, invoking possible interactions of BRMS1 with oncogenic signaling [82]. Importantly, the data from clinical specimens highlights a known, but often under-appreciated, complication that mRNA and protein levels do not necessarily correlate. In the case of BRMS1, we have shown profound regulation of BRMS1 protein at a post-translational level [83]. Therefore, we emphasize caution when interpreting mRNA data.

Since its discovery, mechanistic insights into BRMS1 function have contributed to (but unfortunately somewhat confused) our understanding of how BRMS1 may suppress metastasis. BRMS1 interacts with several large multisubunit mSin3-histone deacetylase (HDAC) enzyme complexes that remove acetyl groups from histone tails changing chromatin structure and correspondingly transcription [84]. Thus, BRMS1 is positioned to regulate a cadre of genes controlling multiple steps in the metastatic cascade. This speculation has been borne out in gene expression profiling [85].

With regard to BRMS1 effects on tumor cell interactions with the tumor microenvironment and extracellular matrix, BRMS1 interacts directly with the nuclear factor-kappa B (NFKB) transcription factor, NFKB consists of a transcription factor heterodimer composed of p50 and p65 subunits maintained in an inactive state in the cytoplasm by physical interaction with the inhibitory regulatory protein IKB. Phosphorylation of I $k$ B by the IKB-alpha kinase (IKK) complex frees $N F K B$ by exposing nuclear localization signals allowing it to translocate and regulate transcription of genes. NFKB is upregulated or constitutively activated in numerous human cancers. One of NFKB's downstream targets is the serine protease, urokinase-type plasminogen activator (uPA). Active uPA cleaves plasminogen in its inactive zymogen state to its active form, plasmin. Active plasmin initiates multiple enzymatic cascades, depending on the microenvironment of a cell. In MDA-MB-231 human breast and C8161.9 human melanoma cell lines, which both exhibit constitutively active $\mathrm{NF} \kappa \mathrm{B}$, expression of BRMS1 inhibited the TNF $\alpha$-induced phosphorylation of IKB and expression of BRMS1 with a concomitant decrease in uPA. Additionally, BRMS1 acting as a co-repressor with HDAC1 has been shown to directly interact with the RelA/p65 subunit of NFKB and cause deacetylation of lysine 310 . The removal of the acetyl groups decreases the transactivation potential of RelA/p65 and, thus, decreases the action of NFKB in non-small cell lung carcinoma (NSCLC) cells [86]. Interestingly, when siRNA was used to knockdown BRMS1, the lack of deacetylation by HDAC1 on RelA/p65 led to NF KB chromatin binding to the promoters of two anti-apoptotic genes, $c I A P 2, B f l-1 / A 1$ suggesting that loss of BRMS1 may lead to a more apoptosis resistant phenotype [86]. 
Recent data confirm that BRMS1-expressing cells are more sensitive to anoikis than their metastatic counterparts [87]. As a result, one would predict reduced survival of BRMS1 cells in the circulation. Phadke et al. [87] and Hedley and colleagues (BRMS1 suppresses breast cancer metastasis in multiple organs by reducing solitary cell survival and inhibiting initiation of growth. B.D. Hedley, K.S. Vaidya, P. A. Phadke, L. MacKenzie, D.W. Dales, C.O. Postenka, I.C. MacDonald, A.F. Chambers, manuscript in preparation) have used green fluorescent protein-tagged tumor cells to quantify the fates of injected tumor cells. BRMS1-expressing human breast carcinoma cells were indeed less likely to reach lungs, bone and liver following direct injection into afferent vessels, consistent to increased sensitivity to anoikis.

Another possible role for BRMS1-mediated interactions with the microenvironment involve data in which BRMS1 expression restored gap junctional intercellular communication via regulation of connexin expression. Connexins are monomeric proteins (typically named by their molecular mass) that form hexamers, known as connexons, that insert into the plasma membrane. Connexons physically connect to connexons on other cells to provide direct communication between cells via transfer of molecules $<1.5 \mathrm{kDa}$, e.g., cAMP, PIP3 and various ions [88]. Connexons are sensitive to levels of $\mathrm{Ca}^{2+}, \mathrm{pH}$, cAMP levels. Loss of gap junction communication between cells occurs during neoplastic progression. Using calcein and lucifer yellow fluorescent dye transfer assays, homotypic gap junction communication was restored in BRMS1-expressing MDA-MB-435 cells. This change was apparently mediated by BRMS1 regulation of individual connexin proteins $[89,90]$. Additionally, in studies examining GJIC between MDA-MB-435 cells and the human osteoblastic cell line hFOB1.19, MDA-MB-435 cells communicated via gap junctions at a greater level with the osteoblasts. BRMS1-expressing cells maintained homotypic communication but lost heterotypic communication with osteoblasts, which may have implications explaining how metastatic breast cancer cells communicate and survive at secondary sites such as bone [91].

The heterotypic GJIC may explain other studies by Phadke et al. [87], who showed that, in addition to increasing sensitivity to anoikis, BRMS1 cells successfully seeding at ectopic sites remained dormant for extended times. At minimum BRMS1 appears to control how tumor cells respond to growth signals differentially (i.e., grow in mammary fat pads but not lungs or bone). Such a difference in growth response can explain why tumor cells expressing metastasis suppressors form primary tumors, but not secondary tumors/metastases.

Another molecule regulated by BRMS1 is the secreted extracellular matrix protein osteopontin (OPN). OPN expression is directly correlated to aggressive behavior in multiple tumor types. When BRMS1 is re-expressed, OPN levels drop by $>75 \%$. Recent data from a collaborative project to Ben Hedley and Ann Chambers lab, restoration of OPN expression in BRMS1-expressing MDA-MB-435 cells restores metastatic competency (Re-expression of Osteopontin in Breast Cancer Metastasis Suppressor 1-Expressing Breast Cancer Cells Restores Metastatic Potential. B.D. Hedley, D.R. Welch, A.L. Allan, W. Al-Katib, D.W. Dales, C.O. Postenka, I.C. MacDonald, A.F. Chambers, manuscript submitted). Clearly, loss of OPN is a downstream effect of BRMS1. Given its secretion into the tumor cell milieu, it is reasonable to assume that it may change the microenvironment. However, direct testing of this hypothesis has not been done.

\section{MKK4}

Evidence for another metastasis suppressor on chromosome 17 was suggested by microcell-mediated chromosome transfer into the highly metastatic AT6.1 rat prostate cancer cell line [92] and subsequent narrowing of the region at a locus away from $\mathrm{Nm} 23$ [93]. Iterative mapping studies identified Mitogen Activated Protein Kinase Kinase 4 (MKK4) as the metastasis suppressor [93-95]. Histologic analyses of human prostate [96] and ovarian [97] cancer samples confirmed an inverse relationship between MKK4 and cancer progression.

Mechanistic insights were first derived from ovarian xenograft models. SKOV3ip.1 ovarian carcinoma cells (which lack endogenous MKK4 expression but maintain all other functional components both up and downstream of MKK4) were inhibited for metastasis by $>90 \%$ when MKK4 was ectopically expressed [97]. Because the molecular and biochemical functions of MKK4 place it in multiple signal transduction pathways by acting as a dual specificity kinase involved in the activation of c-Jun NH2terminal protein kinase (JNK) and p38, the precise actions of how MKK4 inhibits metastasis were still not clearly defined. Both JNK and p38 are activated by stress signals and regulate diverse processes such as apoptosis and cellular proliferation. Subsequent evidence has begun to delineate the multiple actions of MKK4 through JNK and p38 with modulation by MKK7 and MKK6, respectively [98-100].

Examination of MKK4-expressing ovarian carcinoma cells revealed that they seed peritoneal surfaces with high frequency, but do not form macroscopic colonies efficiently [98]. Those observations are consistent with observations that the ratio between p38 signaling and ERK signaling determine proliferative versus dormant states among cancer cells $[101,102]$. The implication is that these pathways are differentially impacted by exogenous signals, however the precise molecules involved have not yet been defined. 


\section{KISS1}

The KISS1 metastasis suppressor was found by subtractive hybridization comparing matched metastasis competent C8161 human melanoma cells to metastasis suppressed chromosome 6-C8161 cell hybrids [103]. KISS1, located on chromosome 1q32, inhibits metastasis in melanoma, breast, and ovarian cancer xenograft models (reviewed in [104]). In clinical samples, KISS1 mRNA levels are inversely correlated to invasiveness in multiple human cancers (reviewed in [104]).

In the C8161 human melanoma xenograft model, cells seeded in the lung, but were unable to proliferate into a macroscopic tumor [105]. The mechanism by which KISS1 prevents colonization remains unclear. However, a major breakthrough toward understanding KISS1 function was made by the work of three independent laboratories showing that KISS1 contains a secretion signal, is preoteolytically processed to make so-called kisspeptins, and secreted [106-108]. Specifically, binding of a 54 amino acid kisspeptin, called metastin, to a G-protein orphan receptor, GPR54, suggested an autocrine feedback loop. Nash and colleagues confirmed that secretion was necessary for the KISS1 anti-metastatic activity. However, they observed something surprising. None of the tumor cells suppressed for metastasis expressed GPR-54, questioning whether the ligand-receptor activation is necessary at all. Although evidence of another receptor was not found (unpublished), the authors have speculated that a tissuespecific paracrine feedback loop may be operational [104].

\section{Summary and Perspectives}

If no other observation were taken into account, the fact that metastasis suppressors allow growth in orthotopic sites but prohibit growth at ectopic sites highlights their central role in how tumor cells interact with the myriad microenvironments encountered during the metastatic cascade. Indeed, the evidence presented in this minireview describe functions for a subset of metastasis suppressors and how each suppressor impacts interactions between tumor cells, matrices and other cell types. What emerges is a picture in which tumor cell expression of specific genes regulate success or failure at each step of the metastatic cascade.

When tumor cells express molecules on their cell surfaces (e.g., E-cadherin) which promote cell-cell adhesion or molecules that prohibit their motility and invasion (e.g., Nm23, TIMPs, SseCKS), they are more apt to remain at the primary tumor. Likewise, molecules that affect recruitment and structural aspects of angiogenic vessels could impact tumor cell dissemination. Still other suppressors reduce cell survival during transit (e.g., caspase-8,
BRMS1, KAI1). We have not even mentioned the multitude of immunogenic molecules that make circulating tumor cells visible to the immune system.

Perhaps most importantly, a growing number of metastasis suppressors, highlighted by KISS1, MKK4, p38 and MKK7 in this review, control the last step of the metastatic process, colonization. When tumor cells arrive at various secondary sites, they find themselves in a milieu of growth promoting and growth inhibitory factors that are different from their site of origin. Depending upon how the cells respond to competing signals, they will either die, remain quiescent or proliferate. It is only when they proliferate that they will become a bona fide metastases. We speculate that a subset of metastasis suppressors will eventually determine in which organs metastases develop. Those molecules would be responsible for the predilection for metastasis observed in many tumor types (e.g., bone metastases from breast and prostate carcinomas).

We find ourselves at the earliest stages of a research endeavor that, at its root, defines cellular responses in complex mixtures of molecules. It has been clear, and the results presented in this short review emphasize, that tumor cells alone do not determine whether metastases develop. It is clear that the response of tumor cells to signals from the many microenvironments through which it traverses to seed and proliferate a secondary site are essential for metastasis. Experiments to elucidate the mechanisms of metastasis are currently relatively basic since they explore only one factor at a time. Significant advances will require a sophisticated and more comprehensive mimicry of the various microenvironments, including growth factors, growth factor ratios, growth inhibitors, matrices and biophysical parameters such as sheer and torsion.

Our objective in writing this review has been to highlight the interactions between tumor cells and various stages of the microenvironment by describing how a selected number of metastasis suppressors control key interactions. We hope that the manner in which we have presented the facts stimulates investigators studying metastasis to incorporate elements of integral cell-cell and cell-matrix interactions.

Acknowledgments Primary research from the Welch lab has been supported by grants from the US National Cancer Institute, Department of Defense, Susan G. Komen for the Cure and the National Foundation for Cancer Research.

\section{References}

1. Wolf $\mathrm{K}, \mathrm{Wu}$ YI, Liu Y et al (2007) Multi-step pericellular proteolysis controls the transition from individual to collective cancer cell invasion. Nat Cell Biol 9:893-904

2. Friedl P, Wolf K (2003) Tumour-cell invasion and migration: Diversity and escape mechanisms. Nat Rev Cancer 3:362-374 
3. Weiss L, Orr FW, Honn KW (1989) Interactions between cancer cells and the microvasculature: A rate regulator for metastasis. Clin Exp Metastasis 7:127-167

4. Weiss L (1990) Metastatic inefficiencyAdv Cancer Res. 54:159211

5. Rennebeck G, Martelli M, Kyprianou N (2005) Anoikis and survival connections in the tumor microenvironment: Is there a role in prostate cancer metastasis? Cancer Res 65:11230-11235

6. Wang SL, El-Deiry WS (2003) TRAIL and apoptosis induction by TNF-family death receptors. Oncogene 22:8628-8633

7. Fidler IJ (1974) Immune stimulation-inhibition of experimental cancer metastasis. Cancer Res 34:491-498

8. Hehlgans S, Haase M, Cordes N (2007) Signalling via integrins: Implications for cell survival and anticancer strategies. Biochim Biophys Acta Rev Cancer 1775:163-180

9. Cavallaro U, Christofori G (2001) Cell adhesion in tumor invasion and metastasis: loss of the glue is not enough. Biochim Biophys Acta Rev Cancer 1552:39-45

10. Pauli BU, Augustin-Voss HG, El-Sabban ME et al (1990) Organpreference of metastasis. The role of endothelial cell adhesion molecules. Cancer Metastasis Rev 9:175-189

11. Nicolson GL (1988) Organ specificity of tumor metastasis: role of preferential adhesion, invasion and growth of malignant cells at specific secondary sites. Cancer Metastasis Rev 7:143-188

12. Hart IR (1982) "Seed and soil" revisited: mechanisms of sitespecific metastasis. Cancer Metastasis Rev 1:5-16

13. Ruffini PA, Morandi P, Cabioglu N et al (2007) Manipulating the chemokine-chemokine receptor network to treat cancer. Cancer 109:2392-2404

14. Naumov GN, MacDonald IC, Chambers AF et al (2001) Solitary cancer cells as a possible source of tumour dormancy? Semin Cancer Biol 11:271-276

15. Al Mehdi AB, Tozawa K, Fisher AB et al (2000) Intravascular origin of metastasis from the proliferation of endothelium-attached tumor cells: a new model for metastasis. Nat Med 6:100-102

16. Townson JL, Chambers AF (2006) Dormancy of solitary metastatic cells. Cell Cycle 5:1744-1750

17. Chambers AF, Groom AC, MacDonald IC (2002) Dissemination and growth of cancer cells in metastatic sites. Nat Rev Cancer 2:563-572

18. Eccles SA, Welch DR (2007) Metastasis: recent discoveries and novel treatment strategies. Lancet 369:1742-1757

19. Welch DR (2007) Do we need to redefine a cancer metastasis and staging definitions? Breast Disease 26:3-12

20. Chambers AF, Matrisian LM (1997) Changing views of the role of matrix metalloproteinases in metastasis. J Natl Cancer Inst $89: 1260-1270$

21. Vaidya KS, Welch DR (2007) Metastasis suppressors and their roles in breast carcinoma. J Mamm Gland Biol Neopl 12:175-190

22. Rinker-Schaeffer CW, O'Keefe JP, Welch DR et al (2006) Metastasis suppressor proteins: Discovery, Molecular mechanisms and Clinical Application. Clin Cancer Res 12:3382-3389

23. Guarino M, Rubino B, Ballabio G (2007) The role of epithelialmesenchymal transition in cancer pathology. Pathology 39:305318

24. Graff JR, Gabrielson E, Fujii H et al (2000) Methylation patterns of the E-cadherin $5^{\prime} \mathrm{CpG}$ island are unstable and reflect the dynamic, heterogeneous loss of E-cadherin expression during metastatic progression. J Biol Chem 275:2727-2732

25. Graff JR, Greenberg VE, Herman JG et al (1998) Distinct patterns of E-cadherin $\mathrm{CpG}$ island methylation in papillary, follicular, Hurthle's cell, and poorly differentiated human thyroid carcinoma. Cancer Res 58:2063-2066

26. Hugo H, Ackland ML, Blick T et al (2007) Epithelialmesenchymal and mesenchymal-epithelial transitions in carcinoma progression. J Cell Physiol 213:374-383
27. Potter JD (2007) Morphogens, morphostats, microarchitecture and malignancy. Nat Rev Cancer 7:464-474

28. Hay ED (2005) The mesenchymal cell, its role in the embryo, and the remarkable signaling mechanisms that create it. Dev Dyn 233:706-720

29. Thiery JP (2002) Epithelial-mesenchymal transitions in tumour progression. Nat Rev Cancer 2:442-454

30. Foty RA, Steinberg MS(2004) Cadherin-mediated cell-cell adhesion and tissue segregation in relation to malignancy. Int $\mathbf{J}$ Dev Biol 48:397-409

31. Kashima T, Nakamura K, Kawaguchi J et al (2003) Overexpression of cadherins suppresses pulmonary metastasis of osteosarcoma in vivo. Int J Cancer 104:147-154

32. Puisieux A, Valsesia-Wittmann S, Ansieau S (2006) A twist for survival and cancer progression. Br J Cancer 94:13-17

33. Yang J, Mani SA, Weinberg RA (2006) Exploring a new twist on tumor metastasis. Cancer Res 66:4549-4552

34. Weaver AM (2006) Invadopodia: specialized cell structures for cancer invasion. Clin Exp Metastasis 23:97-105

35. Christiansen JJ, Rajasekaran AK (2006) Reassessing epithelial to mesenchymal transition as a prerequisite for carcinoma invasion and metastasis. Cancer Res 66:8319-8326

36. Tarin D (2005) The fallacy of epithelial mesenchymal transition in neoplasia Cancer Res 65: 5996-6000

37. Tarin D (2005) Carcinoma invasion and metastasis: A role for epithelial-mesenchymal transition? Response. Cancer Res 65:5995-5995

38. Lin X, Tombler E, Nelson PJ et al (1996) A novel src- and rassuppressed protein kinase $\mathrm{C}$ substrate associated with cytoskeletal architecture. J Biol Chem 271:28430-28438

39. Gelman IH, Lee K, Tombler E et al (1998) Control of cytoskeletal architecture by the src-suppressed $\mathrm{C}$ kinase substrate, SSeCKS. Cell Motil Cytoskelet 41:1-17

40. Xia W, Unger P, Miller L et al (2001) The Src-suppressed C kinase substrate, SSeCKS, is a potential metastasis inhibitor in prostate cancer. Cancer Res 61:5644-5651

41. Lin X, Nelson P, Gelman IH (2000) SSeCKS, a major protein kinase $C$ substrate with tumor suppressor activity, regulates $G$ (1) $>$ S progression by controlling the expression and cellular compartmentalization of cyclin D. Mol Cell Biol 20:7259-7272

42. Xia W, Gelman IH (2002) Mitogen-induced, FAK-dependent tyrosine phosphorylation of the SSeCKS scaffolding protein. Exp Cell Res 277:139-151

43. Steeg PS, Bevilacqua G, Kopper L et al (1988) Evidence for a novel gene associated with low tumor metastatic potential. J Natl Cancer Inst 80:200-204

44. Pozzatti R, Muschel RJ, Williams J et al (1986) Primary rat embryo cells transformed by one or two oncogenes show different metastatic potentials. Science 232:223-227

45. Hartsough MT, Steeg PS (2000) Nm23/nucleoside diphosphate kinase in human cancers. J Bioenerg Biomembranes 32:301308

46. Freije JM, MacDonald NJ, Steeg PS (1998) Nm23 and tumour metastasis: basic and translational advances. Biochem Soc Symp 63:261-271

47. McDermott WG, Boissan M, Lacombe ML et al (2007) Nm23H1 homologs suppress tumor cell motility and anchorage independent growth. Clin Exp Metastasis. doi:10.1007/ s10585-007-9128-0

48. Hailat N, Keim DR, Melhem RF et al (1991) High levels of p19/ nm23 protein in neuroblastoma are associated with advanced stage disease and with $\mathrm{N}-m y c$ gene amplification. J Clin Invest $88: 341-345$

49. Fournier HN, Dupé-Manet S, Bouvard D et al (2002) Integrin cytoplasmic domain-associated protein $1 \alpha$ (ICAP-1 $\alpha)$ interacts directly with the metastasis suppressor $\mathrm{nm} 23-\mathrm{H} 2$, and both 
proteins are targeted to newly formed cell adhesion sites upon integrin engagement. J Biol Chem 277:20895-20902

50. Tee YT, Chen GD, Lin LY et al (2006) Nm23-H1: a metastasisassociated gene. Taiwan J Obstet Gynecol 45:107-113

51. Otsuki Y, Tanaka M, Yoshii S et al (2001) Tumor metastasis suppressor $\mathrm{nm} 23 \mathrm{H} 1$ regulates Rac1 GTPase by interaction with Tiam1. Proc Natl Acad Sci 98:4385-4390

52. Kuppers DA, Lan K, Knight JS et al (2005) Regulation of matrix metalloproteinase 9 expression by Epstein-Barr virus nuclear antigen $3 \mathrm{C}$ and the suppressor of metastasis Nm23-H1. J Virol 79:9714-9724

53. Che G, Chen J, Liu L et al (2006) Transfection of nm23-H1 increased expression of beta-Catenin, E-Cadherin and TIMP-1 and decreased the expression of MMP-2, CD44v6 and VEGF and inhibited the metastatic potential of human non-small cell lung cancer cell line L9981. Neoplasma 53:530-537

54. Horak CE, Lee JH, Elkahloun AG et al (2007) Nm23-H1 suppresses tumor cell motility by down-regulating the lysophosphatidic acid receptor EDG2. Cancer Res 67:7238-7246

55. Kishi J, Tanaka R, Koiwai O et al (1994) Gelatinases and metalloproteinase inhibitor secreted by murine colonic carcinoma cells with differing metastatic potential. Cell Biol Int 18:165170

56. Li H, Fang W, Shi Z (1997) Effects of TIMP-2 gene transfection on biological behaviors of a metastatic human lung carcinoma cell line. Zhonghua Yi Xue Za Zhi 77:652-656

57. Lopez-Otin C, Matrisian LM (2007) Emerging roles of proteases in tumour suppression. Nat Rev Cancer 7:800-808

58. Takahashi C, Sheng Z, Horan TP et al (1998) Regulation of matrix metalloproteinase- 9 and inhibition of tumor invasion by the membrane-anchored glycoprotein RECK. Proc Natl Acad Sci 95:13221-13226

59. Noda M, Oh J, Takahashi R et al (2003) RECK: a novel suppressor of malignancy linking oncogenic signaling to extracellular matrix remodeling. Cancer Metastasis Rev $22: 167-175$

60. Correa TCS, Brohem CA, Winnischofer SMB et al (2006) Downregulation of the RECK-tumor and metastasis suppressor gene in glioma invasiveness. J Cell Biochem 99:156-167

61. Span PN, Sweep CGJF, Manders P et al (2003) Matrix metalloproteinase inhibitor reversion-inducing cysteine-rich protein with Kazal motifs - A prognostic marker for good clinical outcome in human breast carcinoma. Cancer 97:2710-2715

62. Oh J, Seo DW, Diaz T et al (2004) Tissue inhibitors of metalloproteinase 2 inhibits endothelial cell migration through increased expression of RECK. Cancer Res 64:9062-9069

63. Noda M, Takahashi C (2007) Recklessness as a hallmark of aggressive cancer. Cancer Sci 98:1659-1665

64. Chang HC, Cho CY, Hung WC (2006) Silencing of the metastasis suppressor RECK by RAS oncogene is mediated by DNA methyltransferase $3 b$-induced promoter methylation. Cancer Res 66:8413-8420

65. Chang HC, Liu LT, Hung WC (2004) Involvement of histone deacetylation in ras-induced down-regulation of the metastasis suppressor RECK. Cell Signal 16:675-679

66. Liu LT, Chang HC, Chiang LC et al (2003) Histone deacetylase inhibitor up-regulates RECK to inhibit MMP-2 activation and cancer cell invasion. Cancer Res 63:3069-3072

67. Oh J, Takahashi R, Kondo S et al (2001) The membraneanchored MMP inhibitor RECK is a key regulator of extracellular matrix integrity and angiogenesis. Cell 107:789-800

68. Li SL, Gao DL, Zhao ZH et al (2007) Correlation of matrix metalloproteinase suppressor genes RECK, VEGF, and CD105 with angiogenesis and biological behavior in esophageal squamous cell carcinoma. World J Gastroenterol 13:6076-6081
69. Dong JT, Lamb PW, Rinker-Schaeffer CW et al (1995) KAI1, a metastasis suppressor gene for prostate cancer on human chromosome 11p11.2. Science 268:884-886

70. Liu WM, Zhang XA (2006) KAI1/CD82, a tumor metastasis suppressor. Cancer Lett 240:183-194

71. Kauffman EC, Robinson VL, Stadler WM et al (2003) Metastasis suppression: the evolving role of metastasis suppressor genes for regulating cancer cell growth at the secondary site. J Urol 169:1122-1133

72. Jee BK, Park KM, Surendran S et al (2006) KAI1/CD82 suppresses tumor invasion by MMP9 inactivation via TIMP1 up-regulation in the H1299 human lung carcinoma cell line. Biochem Biophys Res Commun 342:655-661

73. Bandyopadhyay S, Zhan R, Chaudhuri A et al (2006) Interaction of KAI1 on tumor cells with DARC on vascular endothelium leads to metastasis suppression. Nat Med 12:933-938

74. Seraj MJ, Samant RS, Verderame MF et al (2000) Functional evidence for a novel human breast carcinoma metastasis suppressor, BRMS1, encoded at chromosome 11q13. Cancer Res 60:2764-2769

75. Shevde LA, Samant RS, Goldberg SF et al (2002) Suppression of human melanoma metastasis by the metastasis suppressor gene, BRMS1. Exp Cell Res 273:229-239

76. Zhang S, Lin QD, DI W (2006) Suppression of human ovarian carcinoma metastasis by the metastasis-suppressor gene, BRMS1. Int J Gynecol Cancer 16:522-531

77. Lombardi G, Di Cristofano C, Capodanno A et al (2006) High level of messenger RNA for BRMS1 in primary breast carcinomas is associated with poor prognosis. Int $\mathrm{J}$ Cancer 120:1169-1178

78. Zhang Z, Yamashita H, Toyama $\mathrm{T}$ et al (2006) Reduced expression of the breast cancer metastasis suppressor $1 \mathrm{mRNA}$ is correlated with poor progress in breast cancer. Clin Cancer Res 12:6410-6414

79. Ohta S, Lai EW, Pang ALY et al (2005) Downregulation of metastasis suppressor genes in malignant pheochromocytoma. Int J Cancer 114:139-143

80. Stark AM, Tongers K, Maass N et al (2004) Reduced metastasissuppressor gene mRNA-expression in breast cancer brain metastases. J Cancer Res Clin Oncol 131:191-198

81. Kelly LM, Buggy Y, Hill A et al (2005) Expression of the breast cancer metastasis suppressor gene, BRMS1, in human breast carcinoma: lack of correlation with metastasis to axillary lymph nodes. Tumor Biol 26:213-216

82. Hicks DG, Yoder BJ, Short S et al (2006) Loss of BRMS1 protein expression predicts reduced disease-free survival in hormone receptor negative and HER2 positive subsets of breast cancer. Clin Cancer Res 12:6702-6708

83. Hurst DR, Mehta A, Moore BP et al (2006) Breast cancer metastasis suppressor 1 (BRMS1) is stabilized by the Hsp90 chaperone. Biochem Biophys Res Commun 348:1429-1435

84. Meehan WJ, Samant RS, Hopper JE et al (2004) Breast cancer metastasis suppressor 1 (BRMS1) forms complexes with retinoblastoma-binding protein 1 (RBP1) and the mSin3 histone deacetylase complex and represses transcription. J Biol Chem 279:1562-1569

85. Champine PJ, Michaelson J, Weimer B et al (2007) Microarray analysis reveals potential mechanisms of BRMS1-mediated metastasis suppression. Clin Exp Metastasis 24:551-565

86. Liu Y, Smith PW, Jones DR (2006) Breast cancer metastasis suppressor 1 functions as a corepressor by enhancing histone deacetylase 1-mediated deacetylation of RelA/p65 and promoting apoptosis. Mol Cell Biol 26:8683-8696

87. Phadke PA, Vaidya KS, Nash KT et al (2008) BRMS1 suppresses breast cancer experimental metastasis to multiple 
organs by inhibiting several steps of the metastatic process. Am J Pathol (in press)

88. Simon AM (1999) Gap junctions: more roles and new structural data. Trends Cell Biol 9:169-170

89. Samant RS, Seraj MJ, Saunders MM et al (2001) Analysis of mechanisms underlying BRMS1 suppression of metastasis. Clin Exp Metastasis 18:683-693

90. Saunders MM, Seraj MJ, Li ZY et al (2001) Breast cancer metastatic potential correlates with a breakdown in homospecific and heterospecific gap junctional intercellular communication. Cancer Res 61:1765-1767

91. Kapoor P, Saunders MM, Li Z et al (2004) Breast cancer metastatic potential: Correlation with increased heterotypic gap junctional intercellular communication between breast cancer cells and osteoblastic cells. Int J Cancer 111:693-697

92. Rinker-Schaeffer CW, Hawkins AL, Ru N et al (1994) Differential suppression of mammary and prostate cancer metastasis by human chromosomes 17 and 11. Cancer Res 54:6249-6256

93. Chekmareva MA, Hollowell CP, Smith RC et al (1997) Localization of prostate cancer metastasis-suppressor activity on human chromosome 17. Prostate 33:271-280

94. Yoshida BA, Dubauskas Z, Chekmareva MA et al (1999) Identification and characterization of candidate prostate cancer metastasis-suppressor genes encoded on human chromosome 17. Cancer Res 59:5483-5487

95. Yoshida BA, Dubauskas Z, Chekmareva MA et al (1999) Mitogen-activated protein kinase kinase $4 /$ stress-activated protein/Erk kinase 1 (MKK4/SEK1), a prostate cancer metastasis suppressor gene encoded by human chromosome 17. Cancer Res 59:5483-5487

96. Kim HL, Van der Griend DJ, Yang X et al (2001) Mitogenactivated protein kinase kinase 4 metastasis suppressor gene expression is inversely related to histological pattern in advancing human prostatic cancers. Cancer Res 61:2833-2837

97. Yamada SD, Hickson JA, Hrobowski Y et al (2002) Mitogenactivated protein kinase kinase 4 (MKK4) acts as a metastasis suppressor gene in human ovarian carcinoma. Cancer Res 62:6717-6723

98. Hickson JA, Huo D, Vander Griend DJ et al (2006) The p38 Kinases MKK4 and MKK6 suppress metastatic colonization in human ovarian carcinoma. Cancer Res 66:2264-2270

99. Vander Griend DJ, Kocherginsky M, Hickson JA et al (2005) Suppression of metastatic colonization by the context-dependent activation of the c-jun NH2-terminal kinase kinases JNKK1/ MKK4 and MKK7. Cancer Res 65:10984-10991

100. Vander Griend DJ, Rinker-Schaeffer CW (2004) A new look at an old problem: the survival and organ-specific growth of metastases. Sci STKE 2004:216pe3

101. Aguirre-Ghiso JA (2006) The problem of cancer dormancy understanding the basic mechanisms and identifying therapeutic opportunities. Cell Cycle 5:1740-1743

102. Aguirre-Ghiso JA, Estrada Y, Liu D et al (2003) ERK(MAPK) activity as a determinant of tumor growth and dormancy; regulation by p38(SAPK). Cancer Res 63:1684-1695

103. Lee J-H, Miele ME, Hicks DJ et al (1996) KiSS-1, a novel human malignant melanoma metastasis-suppressor gene. J Natl Cancer Inst 88:1731-1737

104. Nash KT, Welch DR (2006) The KISS1 metastasis suppressor: mechanistic insights and clinical utility. Front Biosci 11:647-659

105. Nash KT, Phadke PA, Navenot J-M et al (2007) KISS1 metastasis suppressor secretion, multiple organ metastasis suppression, and maintenance of tumor dormancy. J Natl Cancer Inst 99:309-321

106. Kotani M, Detheux M, Vandenbogaerde A et al (2001) The metastasis suppressor gene KiSS-1 encodes kisspeptins, the natural ligands of the orphan $G$ protein-coupled receptor GPR54. J Biol Chem 276:34631-34636

107. Muir AI, Chamberlain L, Elshourbagy NA et al (2001) AXOR12: A novel human $\mathrm{G}$ protein-coupled receptor, activated by the peptide KiSS-1. J Biol Chem 276:28969-28975

108. Ohtaki T, Shintani Y, Honda S et al (2001) Metastasis suppressor gene KiSS1 encodes peptide ligand of a G-protein-coupled receptor. Nature 411:613-617 